Stereotactic and Functional

Neurosurgery
Stereotact Funct Neurosurg 2010;88:11-15

DOI: $\underline{10.1159 / 000260074}$
Received: January 29,2009

Accepted after revision: August 30, 2009

Published online: November 20, 2009

\title{
Electrocorticographic Frequency Alteration Mapping of Speech Cortex during an Awake Craniotomy: Case Report
}

\author{
J. Breshears ${ }^{d} \quad$ M. Sharma ${ }^{a} \quad$ N.R. Anderson ${ }^{a}$ S. Rashid ${ }^{c}$ E.C. Leuthardt ${ }^{a}$ b \\ a Department of Biomedical Engineering, Washington University in St. Louis, and Departments of \\ ${ }^{b}$ Neurological Surgery and ${ }^{\mathrm{C}}$ Neurology, School of Medicine, and ${ }^{\mathrm{d}}$ School of Medicine, Washington University \\ in St. Louis, St. Louis, Mo., USA
}

\section{Key Words}

Electrocorticographic frequency alteration mapping •

Speech cortex · Awake craniotomy

\begin{abstract}
Objective: Traditional electrocortical stimulation (ECS) mapping is limited by the lengthy serial investigation (one location at a time) and the risk of afterdischarges in localizing eloquent cortex. Electrocorticographic frequency alteration mapping (EFAM) allows the parallel investigation of many cortical sites in much less time and with no risk of afterdischarges because of its passive nature. We examined its use with ECS in the context of language mapping during an awake craniotomy for a tumor resection. Clinical Presentation: The patient was a 61-year-old right-handed Caucasian male who presented with headache and mild aphasia. Imaging demonstrated a 3-cm cystic mass in the posterior temporal-parietal lobe. The patient underwent an awake craniotomy for the mapping of his speech cortex and resection of the mass. Intervention: Using a 32-contact electrode array, electrocorticographic signals were recorded from the exposed cortex as the patient participated in a 3-min screening task involving active (patient naming visually presented words) and rest (patient silent) conditions. A spectral comparison of the 2 conditions revealed specific cortical loca-
\end{abstract}

tions associated with activation during speech. The patient was then widely mapped using ECS. Three of 4 sites identified by ECS were also identified passively and in parallel by EFAM, 2 with statistical significance and the third by qualitative inspection. Conclusion: EFAM was technically achieved in an awake craniotomy patient and had good concordance with ECS mapping. Because it poses no risk of afterdischarges and offers substantial time savings, EFAM holds promise for future development as an adjunct intraoperative mapping tool. Additionally, the cortical signals obtained by this modality can be utilized for localization in the presence of a tumor adjacent to the eloquent regions.

Copyright $\odot 2009$ S. Karger AG, Basel

\section{Background}

The established practice of electrical stimulation to create transient lesions in the cortex or evoke motor responses, both extra- and intraoperatively, is the 'gold standard' for delineating cortical areas of eloquent function [1-4]. Its role in minimizing functional loss during neurosurgical resection of seizure foci, tumors and vascular malformations is well documented [5-8]. Specifically, the use of electrocortical stimulation (ECS) to localize speech areas is known to improve functional outcomes [7]. More

\section{KARGER}

(c) 2009 S. Karger AG, Basel

Fax +41613061234 E-Mail karger@karger.ch www.karger.com www.karger.com/sfn
Eric C. Leuthardt, MD

Department of Neurological Surgery, Washington University School of Medicine 660 South Euclid Avenue, Campus Box 8057

St. Louis, MO 63110 (USA)

Tel. +1 314362 8012, Fax +1 314362 2107, E-Mail leuthardte@ nsurg.wustl.edu 

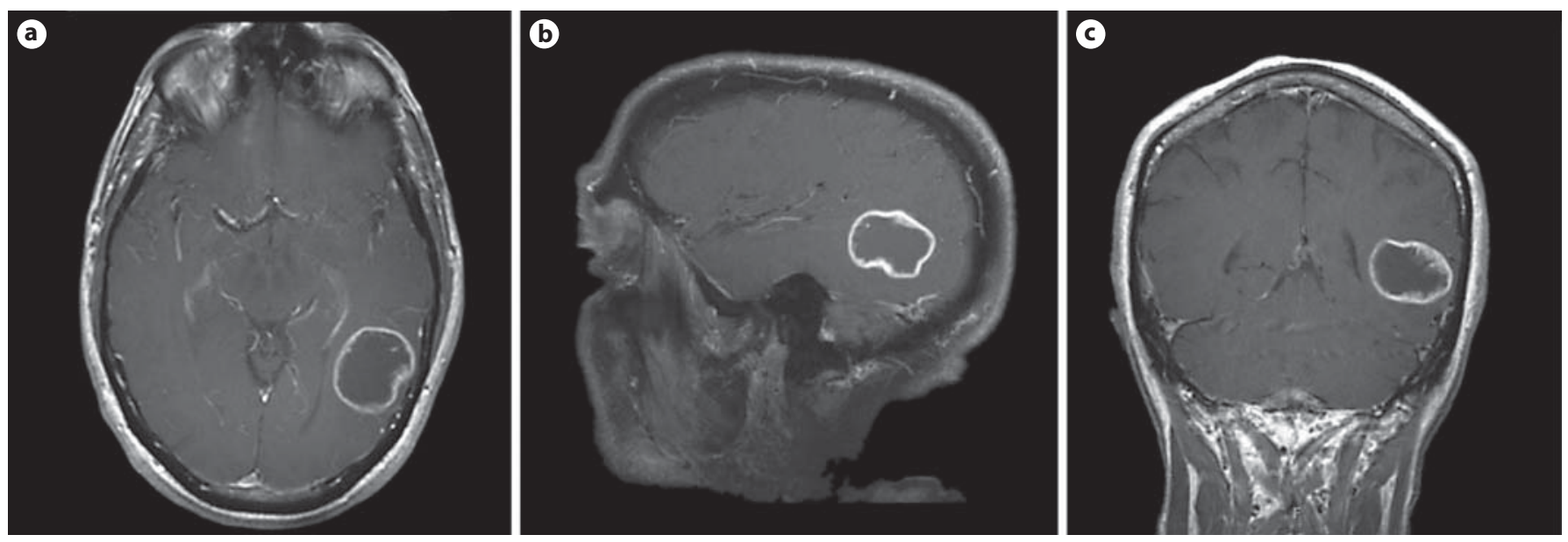

Fig. 1. MRI showing a 3-cm, heterogeneously enhancing, cystic mass in the posterior temporal lobe. Final pathology of the tumor was glioblastoma multiforme.

recently, its use to identify negative speech areas as a guide for resection, in conjunction with tailored craniotomies limiting cortical exposure, has been shown to produce equally favorable functional outcomes [8]. However, ECS has several known limitations. The generation of stimulation afterdischarges in $71 \%$ of mapped patients leads to a risk of seizure or misleading functional responses as a result of distally stimulated cortex [9]. Additionally, inadequate stimulation may leave eloquent cortical areas misidentified. Further, stimulation mapping during awake craniotomies necessitates consistent patient participation, which is not trivial. Given these shortcomings of ECS mapping, the usefulness of electrocorticographic (ECoG) signal analysis as an adjunct approach to cortical mapping has been demonstrated $[10,11]$.

The brain's oscillating electrical signals, recorded extracranially via electroencephalography or subdurally by electrocorticography, are constituted by a broad range of frequencies. Breaking this signal down into its frequency components allows an analysis of how the magnitude, or power, of those components is changing over time. There are several notable distinct frequency bands which are thought to represent different underlying cortical physiologies. Characteristic frequency bands such as mu (8-12 $\mathrm{Hz})$ and beta $(18-26 \mathrm{~Hz})$ are believed to be produced by thalamocortical circuits, and are known to decrease in power during speech, auditory perception, and real or imagined movements [12-14]. This is known as event-related desynchronization. Conversely, the gamma frequency band $(>30 \mathrm{~Hz})$ is thought to be produced by smaller cortical ensembles that demonstrate event-related syn- chronization or increases in power in association with speech, auditory perception, and real or imagined movements [15-19]. These alterations in the frequency power spectrum associated with cortical activation have been investigated as a tool for identifying and delineating regions of the motor cortex [10]. Here, we extend this ECoG frequency alteration mapping (EFAM) technique to the speech cortex in the context of an awake craniotomy.

\section{Case Report}

The patient was a 61-year-old right-handed Caucasian male with a left temporal/parietal lobe lesion scheduled for resection during awake craniotomy (fig. 1). The patient was given monitored anesthesia care with propofol and dexmedetomidine. After the bone flap was removed and the dura was reflected, the patient was taken off propofol and allowed to wake up. A monitor on which language cues were delivered was placed 2-3 feet in front of the patient's eyes. The patient was instructed to say the word that was presented to him on the screen while it was present on the screen (active condition) and to remain quiet during the intervals when no word was presented (rest condition). An $4 \times 8$ electrode array (AdTech, Racine, Wisc., USA) was placed over the exposed cortex (fig. 2a). The electrodes were connected to a data acquisition system which recorded the ECoG signal as the patient was performing the task. The system consisted of optical amplifiers by Guger Technologies and a Dell PC running BCI2000, which presented the stimulus and recorded the ECoG signal simultaneously [20]. BCI2000 is a general-purpose system for data acquisition, stimulus presentation, brain-computer interfacing and brain monitoring [20]. It supports acquisition from a number of hardware devices, can process different types of brain signals, and can feed back the output of that processing to a variety of output devices. In the context of brain mapping, it supports the program- 

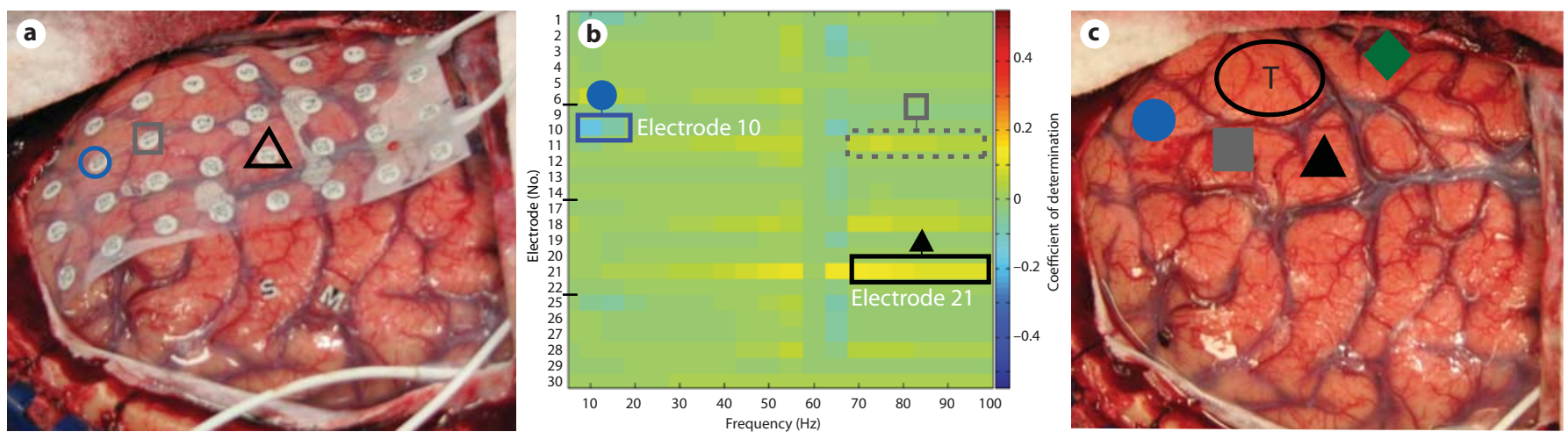

Fig. 2. Relationship between sites identified by passive mapping and ECS. Shapes denote sites identified by EFAM (a, b) and by ECS (c). The corresponding sites between the modalities are shape-matched (circles, squares and triangles). a Grid of 32 electrodes placed over the cortex in preparation for the patient performing the speech task to identify language cortex. The circle and the triangle denote significant sites as activating during speech. The square denotes a qualitatively identified site (see b). b Plot of the coefficient of determination $\left(\mathrm{r}^{2}\right)$ for each frequency across all electrodes. A notable decrease in low frequency amplitude $(5-20 \mathrm{~Hz})$ is identified at the location of electrode 10 (circle; $\left.r^{2}=0.174 ; p=0.01\right)$. Similarly, notable increases in high frequency amplitude $(75-100 \mathrm{~Hz})$ were identified qualitatively at the loca- tions of electrode 11 (dashed square; $\mathrm{r}^{2}=0.074 ; \mathrm{p}=0.1$ ), electrode $18\left(\mathrm{r}^{2}=0.074 ; \mathrm{p}=0.1\right)$ and electrode $28\left(\mathrm{r}^{2}=0.047 ; \mathrm{p}=0.2\right)$, and with statistical significance at electrode 21 (triangle; $\mathrm{r}^{2}=0.143$; $\mathrm{p}=0.02$ ). These locations indicate the probable candidate sites for speech cortex. Electrodes 18 and 28 did not correspond to positive identification by ECS. Electrodes 7, 8, 15, 16, 23, 24, 31 and 32 are not included because they were not in physical contact with the cortex (see a). c All solid shapes denote locations identified as speech cortex by ECS mapping. The circle and the triangle correspond to significant sites identified by EFAM. The square corresponds to a location (electrode 11) identified qualitatively by EFAM. The diamond denotes a site not identified by EFAM. T = Tumor location. mable presentation of auditory/visual stimuli and receipt of device input. BCI2000 associates the timing of these stimuli with the recorded ECoG signals, which facilitates time-locked analyses.

After a trial run, 3 min of data (36 trials) were collected while the patient performed the speech task. The ECoG signal was converted to the spectral domain and the active condition compared to the rest condition. The degree to which the amplitude, or power, change correlated to the given condition was measured by the $r^{2}$, known as the coefficient of determination. For more detail of the signal analysis protocol, please see the previously published protocol in Leuthardt et al. [10]. A plot of the coefficients of determination for each frequency bin and across all electrodes was generated (fig. 2b). Those electrode sites that produced significant amplitude changes at a given frequency were identified intraoperatively by qualitative inspection of figure $2 \mathrm{~b}$. Five electrodes were initially identified qualitatively as potential candidates for speech cortex (electrodes 10, 11, 18, 21 and 28); of these, electrodes 10,11 and 21 demonstrated the strongest activations on qualitative inspection. Postoperatively, the conversion of $r^{2}$ to a $p$ value, using the generalized Fisher method, was done to determine statistical significance. In postoperative analysis, electrode 10 was identified as having a significant coefficient of determination $(\mathrm{p}=0.01)$ at low frequencies $(5-20 \mathrm{~Hz})$ consistent with event-related desynchronization, or a decrease in amplitude, at that frequency band. In electrode $21(\mathrm{p}=0.02)$, power increases (also known as event-related synchronizations) in the higher frequencies $(75-100 \mathrm{~Hz})$ were found to be statistically significant. Electrodes 11,18 and 28 were not found to be significant in postoperative analysis.
After the qualitative identification of potential speech sites from EFAM, the patient's cortex was then widely mapped by ECS, with stimulation currents ranging from 2 to $8 \mathrm{~mA}$. The total time required for ECS was approximately 30-40 min. There were 2 episodes of afterdischarges requiring cold irrigation of the brain surface. When stimulated, the cortical sites beneath electrodes 10 , 11 and 21 induced a speech arrest. There was a substantial overlap in sites identified by EFAM and by ECS for language. Of note, however, there were some functional sites that did not overlap. One site identified by ECS on the middle temporal gyrus was not identified by EFAM (shown as a diamond in fig. 2c). Additionally, there were 2 qualitatively identified electrode sites that were not associated with speech arrest by stimulation; these included electrodes $18(\mathrm{p}=0.1)$ and $28(\mathrm{p}=0.2)$. Subsequent to the identification of language sites, a gross total resection of the tumor was achieved. The resection was guided by the ECS mapping although all the sites identified by EFAM and ECS were spared. The patient's language was receptively and expressively fluent after the operation. The final pathology of the tumor was glioblastoma multiforme.

\section{Discussion}

EFAM has been shown to be an effective adjunct to cortical stimulation for mapping the motor cortex in the extraoperative setting [10]. In this case, we have extended 
these techniques to the application of language localization during an awake craniotomy for a tumor resection. We demonstrate here for the first time that the EFAM methodology can localize regions of cortex involved with receptive language. This, additionally, is the first time it has been done in the setting of an awake craniotomy. In contrast to extraoperative mapping where the cortical tissue is not distorted or infiltrated, we have shown the cortical signals to be reliable in identifying functional sites in close proximity to an intrinsic brain tumor. When compared to localization by ECS, there was a strong concordance in functional sites identified by both methodologies. Of note, the performance of EFAM mapping (cognitive paradigm plus time for signal analysis) was brief and not associated with afterdischarges when compared with ECS mapping.

In much the same way that somatosensory-evoked potentials provide passive information on the localization of motor cortex, so too can the EFAM method provide powerful screening information that can aid in the more efficient localization of language. Thus far, a passive screen does not exist for language as it does for motor mapping. Since a two-dimensional electrode array is recorded simultaneously, multiple sites spanning the cortical surface can be interrogated in parallel. Thus, a 'single pass' is all that is required to assess a large region of cortex (vs. serial site-by-site interrogation by ECS). Additionally, because localization is achieved through passive recording and signal analysis, an identification of functional regions does not carry the risk of afterdischarges associated with ECS. These features of large cortical coverage, parallel recording and absence of afterdischarges thus provide notable time savings in providing a preliminary localization of language cortex. This time-efficient and passive approach is of particular importance when considering an awake craniotomy where patient participation, patient tolerance and induced seizures can make a substantial difference in whether a functional site is located or not.

It is worth noting that, although there was a substantial overlap between the sites identified by EFAM and ECS, there were also regions that did not overlap. There was a site that was identified by ECS that was not identified by EFAM, and there were sites identified by EFAM not associated with speech arrest by ECS. The fact that EFAM does not localize each ECS site underlines that the technique is not currently a standalone method for language localization. The lack of a complete localization may in part reflect the specificity of the EFAM technique at identifying cortical networks particular to a given cog- nitive task. An increased sensitivity (identifying every ECS site) may result if the cognitive task is more varied (reading words as well as naming objects, etc.) and, thus, increasing the number of cortical networks engaged. Currently, the clinical significance is unclear for sites that show changes identified by EFAM but are not associated with a speech arrest by ECS. One hypothesis is that such sites represent other parallel nonessential processes that occur in conjunction with the articulation of speech but are not essential or specific to its implementation, such as attention or memory. Their nonessential nature could explain why they are not identified by ECS. A larger case series will be required to formally assess the clinical significance of these sites.

In conclusion, EFAM is technically possible in awake craniotomy patients and holds promise for development as an adjunct method to aid in defining speech cortex by ECS. The passive and parallel interrogation of the cortex by EFAM offer notable time efficiency advantages that further support its development. A larger patient population will be required to definitively assess the utility and safety of EFAM.

\section{Acknowledgments}

The authors received a James S. McDonnell Higher Brain Function grant as well as grants from the following institutions: the US Department of Defense, the US National Institute of Neurological Disorders and Stroke (NINDS) and the Children's Discovery Institute, St. Louis Children's Hospital, Washington University School of Medicine, USA.

References

Breshears/Sharma/Anderson/Rashid/ Leuthardt 
4 Keles GE, Lundin DA, Lamborn KR, Chang EF, Ojemann G, Berger MS: Intraoperative subcortical stimulation mapping for hemispherical perirolandic gliomas located within or adjacent to the descending motor pathways: evaluation of morbidity and assessment of functional outcome in 294 patients. J Neurosurg 2004;100:369-375.

$\checkmark 5$ Berger MS, Kincaid J, Ojemann GA, Lettich E: Brain mapping techniques to maximize resection, safety, and seizure control in children with brain tumors. Neurosurgery 1989; 25:786-792.

6 Burchiel KJ, Clarke H, Ojemann GA, Dacey RG, Winn HR: Use of stimulation mapping and corticography in the excision of arteriovenous malformations in sensorimotor and language-related neocortex. Neurosurgery 1989;24:322-327.

$\checkmark 7$ Haglund MM, Berger MS, Shamseldin M, Lettich E, Ojemann GA: Cortical localization of temporal lobe language sites in patients with gliomas. Neurosurgery 1994;34: 567-576.

8 Nader S, Zaman M, Berger M: Functional outcome after language mapping for glioma resection. N Engl J Med 2008;358:18-27.

-9 Pouratian N, Cannestra AF, Bookheimer SY, Martin NA, Toga AW: Variability of intraoperative electrocortical stimulation mapping parameters across and within individuals. J Neurosurg 2004;101:458-466.
10 Leuthardt EC, Miller K, Anderson NR, Schalk G, Dowling J, Miller J, Moran DW Ojemann JG: Electrocorticographic frequency alteration mapping: a clinical technique for mapping the motor cortex. Neurosurgery 2007;60(suppl 2):260-270.

11 Ikeda A, Miyamoto S, Shibasaki H: Cortical motor mapping in epilepsy patients: information from subdural electrodes in presurgical evaluation. Epilepsia 2002;43 (suppl 9): 56-60.

12 Levine SP, Huggins JE, BeMent SL, Kushwaha RK, Schuh LA, Passaro EA, Rohde MM, Ross DA: Identification of electrocorticogram patterns as the basis for a direct brain interface. J Clin Neurophysiol 1999;16: 439-447.

-13 Pfurtscheller G, Graimann B, Huggins JE, Levine SP, Schuh LA: Spatiotemporal patterns of beta desynchronization and gamma synchronization in corticographic data during self-paced movement. Clin Neurophysiol 2003;114:1226-1236.

14 Rohde MM, BeMent SL, Huggins JE, Levine SP, Kushwaha RK, Schuh LA: Quality estimation of subdurally recorded, event-related potentials based on signal-to-noise ratio. IEEE Trans Biomed Eng 2002;49:31-40.
5 Crone NE, Miglioretti DL, Gordon B, Lesser RP: Functional mapping of human sensorimotor cortex with electrocorticographic spectral analysis. 2. Event-related synchronization in the gamma band. Brain 1998;121: 2301-2315.

16 Leuthardt EC, Schalk G, Wolpaw JR, Ojemann JG, Moran DW: A brain-computer interface using electrocorticographic signals in humans. J Neural Eng 2004;1:63-71.

17 Lopes da Silva FH, Pfurtscheller G (eds): Event-Related Desynchronization. Handbook of Electroencephalography and Clinical Neurophysiology. Amsterdam, Elsevier, 1999.

18 Crone NE, Boatman D, Gordon B, Hao L: Induced electrocorticographic gamma activity during auditory perception. Brazier Awardwinning article 2001. Clin Neurophysiol 2001;112:565-582.

19 Crone NE, Hao L, Hart J Jr, Boatman D, Lesser RP, Irizarry R, Gordon B: Electrocorticographic gamma activity during word production in spoken and sign language. Neurology 2001;57:2045-2053.

20 Schalk G, McFarland DJ, Hinterberger T, Birbaumer N, Wolpaw JR: BCI2000: a general-purpose brain-computer interface (BCI) system. IEEE Trans Biomed Eng 2004; 51:1034-1043. 